\title{
VÍDEOS DIDÁCTICOS DE FÍSICA Y QUÍMICA
}

\author{
MAS i PUJADAS, F., ${ }^{1}$ PANIAGUA VALLE, J.C. ${ }^{1}$ VILASECA i FONT, E. ${ }^{1}$ y BARBOSA TORRALBO, J. ${ }^{2(2)}$ \\ 1. Departament de Química Física, Divisió de Ciències Experimentals i Maternàtiques, Universitat de Barce- \\ lona. C/ Martí i Franquès, 1. 08028 Barcelona. \\ 2. Departament de Química Analítica, Divisió de Ciències Experimentals i Matemàtiques, Universitat de \\ Barcelona. C/ Martí i Franquès, 1. 08028 Barcelona.
}

\section{SUMMARY}

The spectacular development of audiovisual techniques in the last few years has provided a wealth of pedagogical resources which are clearly underexploited. The aim of this work was to improve this situation by making available resources known to teachers. For this purpose we compiled a catalogue of video tapes and films of use in teaching Physics and Chemistry to university students. The catalogue is available in two formats: as a printed list and as an easytowuse computer-retrievable hypertext.

\section{INTRODUCCIÓN}

El desarrollo vertiginoso de la tecnología audiovisual e informática producido durante las últimas décadas ha permitido una notable diversificación de las herramientas utilizables por el enseñante para desempeñar su labor (Bartolomé 1989), lo que ha provocado efectos sobre el sistema global de la enseñanza y sobre el enfoque de la didáctica (Barbosa 1988). Dentro de las técnicas audiovisuales, el vídeo es, sin duda, la que ha experimentado una evolución más acusada en los últimos años, habien-' do traspasado la barrera de la televisión profesional para convertirse en un instrumento de uso doméstico muy generalizado.

En el campo que nos atañe, que abarca la docencia de la Física y la Química a nivel universitario, el vídeo presenta aplicaciones tan interesantes como puede ser la introducción al alumno en las manipulaciones y operaciones básicas en un laboratorio (Barbosa 1989), o bien, mostrar a grupos relativamente numerosos de alumnos la manera correcta de realizar medidas en Química y la correcta interpretación de los resultados (Barbosa et al. 1988), 10 cual ilustra al alumno sobre la importancia que tienen para el avance de la Química Ios razonamientos lógicos realizados sobre la base de resultados de medidas experimentales, y le transmite una faceta artística de aquella disciplina, ya que las medidas exigen un trabajo bien hecho como en toda obra de artesanía.

Noes fácil evaluar la eficacia formativa del uso del vídeo en la Enseñanza Superior. Estudios en este sentido, acompañados de sondeos sobre la aceptación del vídeo por parte del alumnado han sido llevados a cabo en la Universidad de Barcelona en disciplinas tan diversas como Derecho, Pedagogía, Química, Biología y Farmacia (Barbosa y Bartolomé 1990) con resultados altamente significativos; el $85 \%$ de los alumnos consideran que los vídeos han sido convenientes globalmente para su formación, o bien, el $93 \%$ recomendarían que se volvieran a utilizar los vídeos en el curso posterior. Sin embargo, el estudio realizado sobre si el vídeo es un recurso utilizado en la enseñanza universitaria puso también de manifiesto que este medio está aún poco introducido en Ia Universidad de Barcelona, si bien el $88 \%$ del alumnaco está muy a favor de disponer de videotecas que permitan un visionado individualizado. Fueron, en gran parte, las conclusiones de este trabajo las que nos llevaron a plantear la confección de un catálogo de vídeos y películas didácticos a fin de facilitar la adquisición de vídeos por parte de las bibliotecas universitarias y de dar facilidades tanto al profesorado como al alumnado para el 
uso de este medio en aquellos campos donde presenta mayores ventajas su utilización (Demal 1988), siguiendo las sugerencias que para su uso han realizado diversos autores ('Tosi 1984, Mallas 1985).

El principal obstáculo con que tropieza el interesado en la utilización de vídeos educativos es la dispersión que presenta la información pertinente debido al elevado número de entidades productoras y distribuidoras de material audiovisual existentes. El objetivo principal del catálogo que aquí presentamos es fácilitar el acceso a dicha información en el ámbito de la enseñanza de la Física y de la Química a nivel universitario. Restringir el contenido de vídeos y películas a esta porción de las Ciencias Experimentales ha permitido efectuar un seguimiento bastante exhaustivo del material disponible en la uctualidad; por otra parte, la versión informatizada del catálogo permite una fácil actualización periódica del mismo. Fn el catálogo se han contemplado no sólo los temas propios de Física y Química, sino también temas interdisciplinarios entre ambas disciplinas y otros de campos afines, englobados bajo los epígrafes de "Ciencias de la Tierra y del Espacio" y «Ciencias de la Vida». También se incluye una sección de «Generalidades» donde se recogen vídeos y películas sobre temas generales relacionados con la Física y la Química, no necesariamente orientados a la enseñanza, así como otra sección dedicada a «Ciencias exactas y de la Computación", herramientas indispensables para el desarrollo de la K'́sica y de la Química. Esta estructuración permite, por otra parte, una fácil ampliación del catálogo a todo el ámbito de las Ciencias Experimentales y Matemáticas.

En su versión actual, el catálogo incluye un total de 923 artículos entre vídeos y películas, seleccionados entre Ias ofertas de 20 distribuidoras. Somos conscientes que el catálogo no es completo, pero creemos que puede facilitar considerablemente el acceso a la información sobre material audiovisual de interés universitario disponible. Por otra parte, desearíamos que el esfuerzo invertido en la confección de este catálogo sirviera, además, para atraer la atención de las autoridades académicas sobre el tema y conseguir que se dediquen los medios materiales y humanos necesarios para dotar a la universidad de recursos audiovisuales adecuados.

\section{Descripción del catálogo}

Este catálogo se ha elaborado en dos formas de presentación, una escrita, en forma de libro (Vilaseca et al. 1989), y la otra informatizada ${ }^{4}$ (Paniagua et al. 1989). Esta última es una base de datos presentada en forma de hipertexto que funciona bajo el entorno Hypercard del sistema Apple Macintosh. Si bien la versión escrita incorpora diversos índices que simplifican notablemente su consulta, la versión informatizada es de muy fácit utilización y permite una gran diversidad de rutas de búsqueda, lo cual redunda en una mayor rapidez de consulta y facilita la obtención de información sobre temas afines a uno dado, la búsqueda por palabras clave extensiva a distintos tenas, etc. El manejo de esta versión se efectúa íntegramente por medio de carteles enmarcados sobre la panta-
Figura I

Diagrama de flujo que indica las conexiones entre los 8 tipos de pantalla de la versión informatizada del catálogo.

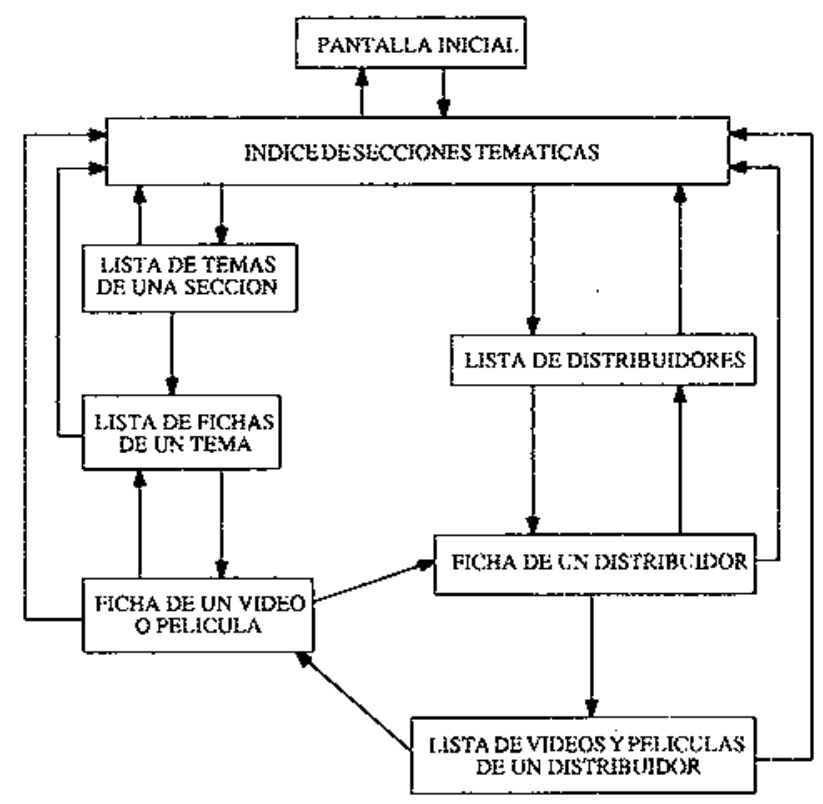

Ila que funcionan a modo de «botones» accionables con la ayuda del ratón, no siendo preciso escribir ninguna instrucción a través del teclado. Por último, el catálogo informatizado está diseñado de forma que sea fácilmente actualizable y ampliable

En la figura 1 se ha representado un diagrama de flujo de las posibles secuencias de consulta realizables. Obsérvese que es altamente interactivo y se aparta de la concepción clásica de diagrama secuencial o ramificado, permitiendo diversas referencias cruzadas. En la figura 2 se muestran, a título de ejemplo, las sucesivas pantallas de una posible secuencia de búsqueda de información.

La clasificación principal se ha hecho por materias, agrupándose éstas en pocas secciones que representan ámbitos de la Ciencia bien establecidos. La relación de temas en cada sección es extensa pero no exhaustiva y responde, en parte, al material audiovisual recogido. Con objeto de facilitar la consulta, y dado que la mayor parte de material recogido no presenta un grado de especifidad elevado, no se han considerado distintos niveles de clasificación dentro de cada sección. İn la tabla I se relacionan las secciones y los temas contemplados en el catálogo, indicándose, entre paréntesis, el número de vídeos y películas pertenecientes a cada tema.

La división entre las secciones de Física y Química no es, en absoluto, estricta, y muchos temas interdisciplinarios, como son la Cinética de las Reacciones Químicas y la Electroquímica, se han incluido bajo el epígrafe de Química Física. Atendiendo las recomendaciones de la UNESCO, se ha considerado una sección de Ciencias de la Tierra y del Espacio independiente de la de Física (cn 
Figura 2

Fjemplo de sccuencia de pantallas que corresponde a una bisqueda de información en la que se han accionado los siguientes botones: "Empezar", Quimica", Química Física", "Ficha uno de estos productos", "Distribuidor" y "Lista de productos de este distribuidor" (pantalla inferior izquierda) o "Lista de distribuidores" (pantalla inferior derecha).

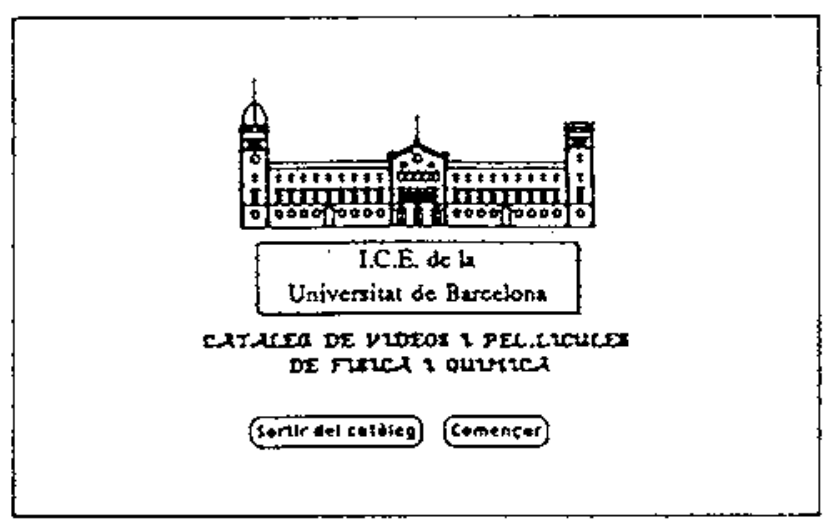

guturics

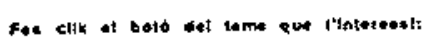

Gerimicotletes

Inden de temas
0888888888888888888888888888889

\section{Jeme: Qulmirs ralse}

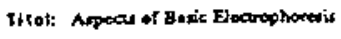

any: 1087

turade: $30 \mathrm{~min}$.

Autart-1]: 2. Winilekt, D. Karmm

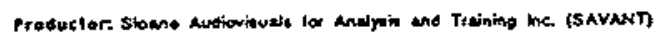

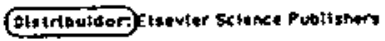

observaclons: $5470, \infty$ \{10as\}

Temas atina

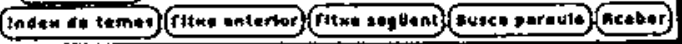

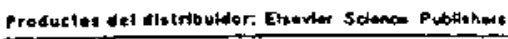

\section{1: Anapits of Enxymes}

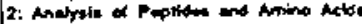

3: Bacte Enxymolions

6: Chitowl Enrymotory

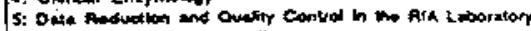

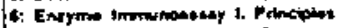

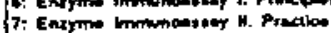

7: Enxymo Imminang

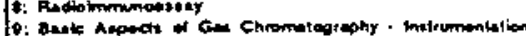

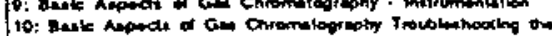

10: Deste neopdo

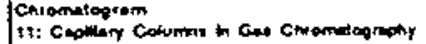

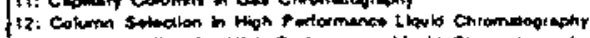

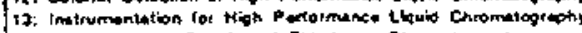

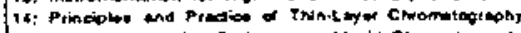

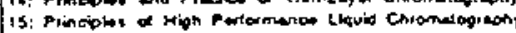

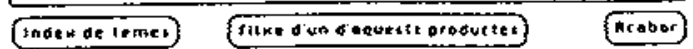

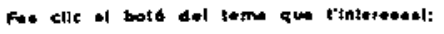

\section{Generittets}

Clenstes enected ide fe cemputecio

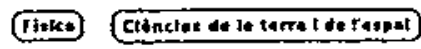

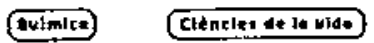

Acoter

Lhtik de Nirtribsldert

Iame?

Proeucter tet tomu: Outmies rinice

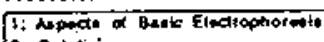

2: Centatis is

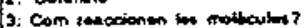

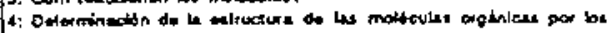

5: Endrollal, Industrisiet

5: Exwotiosuintice

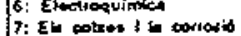

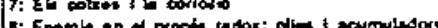

D: Enorgia I Estac OWimic

10: Ephixil y auduon onkpenas

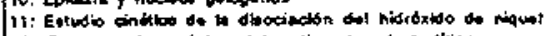

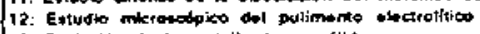

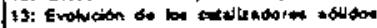

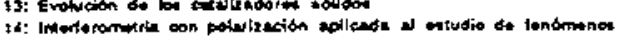

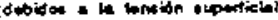

\begin{tabular}{|c|c|c|}
\hline Intex \&\& temes & flime d'un dequeat producles & nceber \\
\hline
\end{tabular}

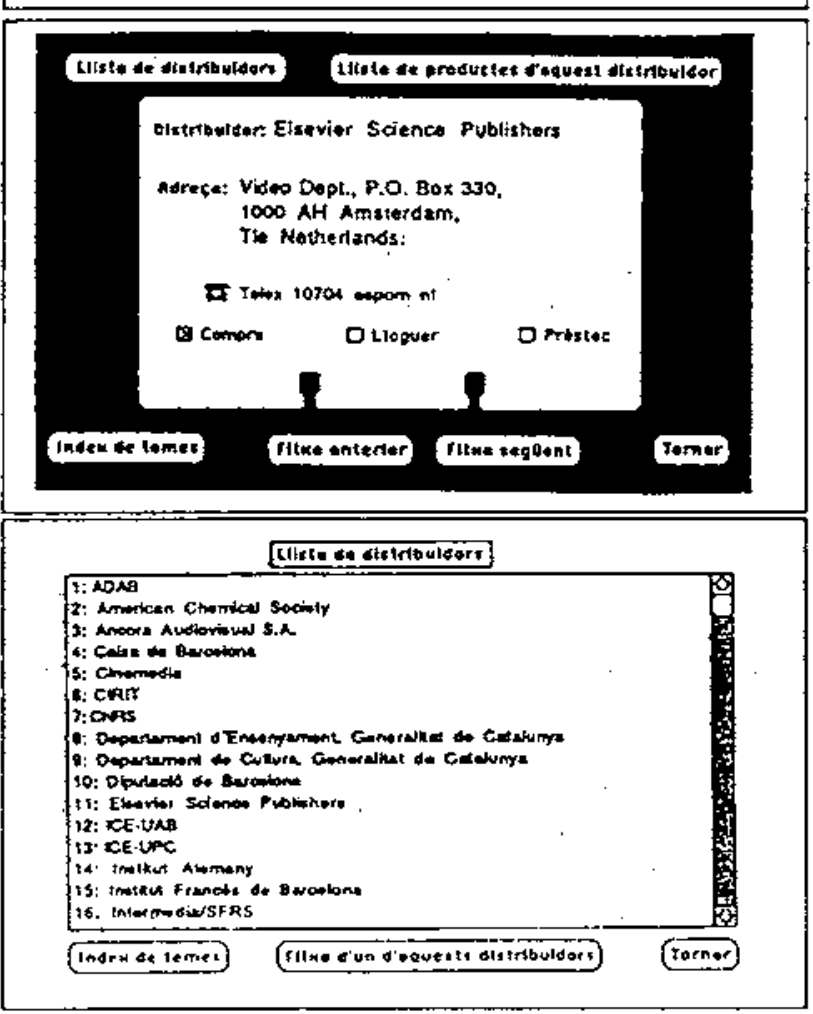




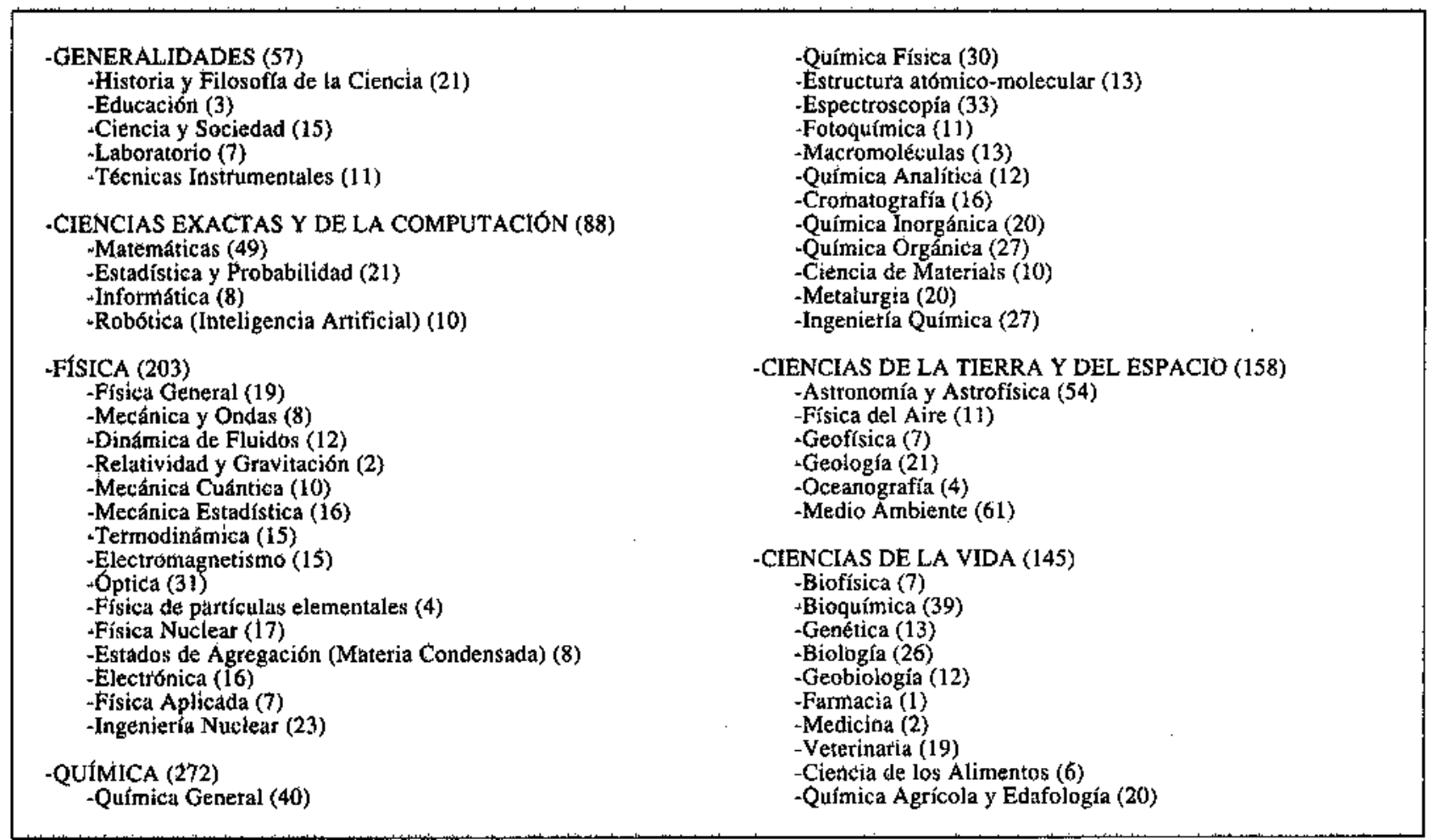

la que se han incluido la Astronomía y la Astrofísica), y una sección de Ciencias de la Vida, en la que los temas de Farmacia, Medicina y Veterinaria tienen, en la ver* sión actual, una representación simbólica. Los temas ambientales y de la bioesfera, debido a su extrema importancia y a la gran cantidad de vídeos y películas que existen sobre ellos, se han clasificado en tres grupos: Oceanografía y Medio Ambiente dentro de la sección de
Ciencias de la Tierra, y Geobiología dentro de Ciencias de la Vida, incluyéndose en este último grupo los vídeos y películas que tratan de la relación de la vida con el planeta Tierra (teoría de Gaia, por ejemplo).

En la tabla II se relacionan, por orden alfabético, las distribuidoras consultadas.
1. ADAB (Distribuidora del ICE de la Universitat de Barcelona)

2. AMERICAN CHEMICAL SOCIETY (Washigton)

3. ANCORA AUDIOVISUAL, S.A. (Distribuidora de la Open University, London)

4. CAIXA DE BARCELONA (Obra Social, Barcelona)

5. CINEMEDIA (Barcelona)

6. CIRIT (Comissio Interdepertamental de Recerca i Innovació Tecnoldgica de a Generalitat de Catalunya, Barcelona)

7. CNRS (Centre National de Recherche Scientifique, París)

8. DEPARTAMENT D'ENSENYAMENT DE LA GENERALITAT DE CATALUNYA

9. DEPARTAMENT DE CULTURA DE LA GENERALITAT DE CATALUNYA

10. DIPUTACIÓ DE BARCELONA
11. ELSEVIER SCIENCE PUBLISHERS (Amsterdam)

12. ICE-UAB (Universitat Aut’noma de Barcelona, Bellaterra)

13. ICE-UPC (Universitat Politècnica de Catalunya, Barcelona)

14. INSTITUTO ALEMAN (Barcelona)

15. INSTITUTO FRANCÉS (Barcelona)

16. INTERMEDIA / SFRS (Direction Générale des Relations Culturalles Scientifiques et Tecniques, París)

17. Mercè DURFORT (Unidad de Bìologra Celular, Facultad de Biolog(a-UB, Barcelona)

18. MUSEO DE LA CIENCIA (Obra Social de La Caixa, Barcelona)

19. PUBLICACIONS DE LA UNIVERSITAT DE BARCELO. NA)

20. SERVEIS DE CULTURA POPULAR (Barcelona) 
En la versión impresa la información de cada vídeo y película se da en forma de ficha, donde se especifican sus características más importantes. La ficha consta de un apartado de observaciones donde se incluyen ciertas aclaraciones sobre su contenido y/o su formato, y se indica si está o no disponible en la videoteca de la

\section{NOTAS}

(1) Trabajo financiado por el Institut de Ciències de l'Educació de la Universitat de Barcelona.

(2) A quien debe dirigirse la correspondencia.
División de Ciencias Experimentales y Matemáticas.

Ambas versiones del catálogo pueden ser consultadas en la biblioteca de Física y Química de la División de Ciencias Experimentales y Matemáticas de la Universi. dad de Barcelona.

(3) Se ha editado un breve resumen del catálogo, que puede ser enviado previa solicitud.

(4) Se puede solicitar la versión informatizada mediante el envío de un disco de 3,5" (doble cara-doble densidad).

\section{REFERENCIAS BIBLIOGRÁFICAS}

BARBOSA, J, 1988. Le video, un instrument pour l'enseignement, Pédagogiques, 8, pp. 421-422.

BARBOSA, J., BOSCH, E., CASASSAS, E., GALCERAN, M.T. y PRAT, M.D., 1988. Teaching of measurement in chemistry through videotapes, Analusis, 16, pp. 201-202.

BARBOSA, J., 1989. Les video comme instrument didactique. Manipulations et operations de base dans un laboratoire chimique, Assempe, 27, pp. 18.

BARBOSA, J. y BARTOLOMÉ, A., 1990. La video, une ressource adaptée à I'enseignement universitaire, Pédago. giques, en prensa.

BARTOLOMÉ, A., 1989. Nuevas tecnologías y enseñanza. (Grao: Barcelona).
DEMAL, J., 1988. Les video au service de l'enseignement universitaire, Le Devoir, 23, p. 5 .

MALLAS, S: 1985.Video y Enseñanza. Universitat de Barcelona.

PANIAGUA, J,C., VILASECA, E. y MAS, F., 1989. Catdleg de Vídeos iPel.licules de Física iQuímica (versió informatizada en Hypercard). (Videoteca de la Biblioteca de Física y Química de la Universitat de Barcelona: Barcelona).

TOSI, V, 1984. How to make scientific advantages for research?, Teaching Popularization, Unesco, París.

VILASECA, E., MAS, F. y PANIAGUA, J.C., 1989. Cataleg de Videos i Pellícules de Física i Química, (ICE-Publicacions de la Universitat de Barcelona: Barcelona), 